\title{
Memristive Behavior of Mixed Oxide Nanocrystal
}

\section{Assemblies}

Zimu Zhou ${ }^{1}$, Pedro López-Domínguez ${ }^{2}$, Muhammad Abdullah ${ }^{3}$, Dylan M. Barber ${ }^{4}$, Xiangxi

Meng $^{5}$, Jieun Park ${ }^{1}$ Isabel Van Driessche ${ }^{2}$, Jessica D. Schiffman ${ }^{5}$, Alfred J. Crosby ${ }^{4}$, Kevin R.

Kittilstved ${ }^{3}$, Stephen S. Nonnenmann ${ }^{1 *}$

${ }^{1}$ Department of Mechanical and Industrial Engineering, University of Massachusetts-Amherst, Amherst, Massachusetts 01003, United States

${ }^{2}$ Department of Chemistry, Ghent University, Ghent, Belgium

${ }^{3}$ Department of Chemistry, University of Massachusetts-Amherst, Amherst, Massachusetts 01003, United States

4 Department of Polymer Science and Engineering, University of Massachusetts-Amherst, Amherst, Massachusetts 01003, United States

5 Department of Chemical Engineering, University of Massachusetts-Amherst, Amherst, Massachusetts 01003, United States

KEYWORDS: solution-processed, resistive switching, memristor, nanocrystals, perovskites *corresponding author: ssn@umass.edu 


\begin{abstract}
Recent advances in memristive nanocrystal assemblies leverage controllable colloidal chemistry to induce a broad range of defect-mediated electrochemical reactions, switching phenomena, and modulate active parameters. The sample geometry of virtually all resistive switching studies involves thin film layers comprising monomodal diameter nanocrystals. Here we explore the evolution of bipolar and threshold resistive switching across highly-ordered, solution-processed nanoribbon assemblies and mixtures comprising $\mathrm{BaZrO}_{3}(\mathrm{BZO})$ and $\mathrm{SrZrO}_{3}$ (SZO) nanocrystals. The effects of nanocrystal size, packing density, and A-site substitution on operating voltage $\left(V_{S E T} ; V_{T H}\right)$ and switching mechanism were studied through a systematic comparison of nanoribbon heterogeneity (i.e., BZO-BZO vs. BZO-SZO) and monomodal vs bimodal size distributions (i.e., small-small; small-large). Analysis of the current-voltage response confirm that tip-induced, trap-mediated space-charge-limited current and trap-assisted tunneling processes drive the low resistance and high resistance states, respectively. Our results demonstrate that both smaller nanocrystals and heavier alkaline earth substitution decrease the onset voltage and improve stability and state retention of monomodal assemblies and bimodal nanocrystal mixtures, thus providing a base correlation that informs fabrication of solution-processed, memristive nanocrystal assemblies.
\end{abstract}

\title{
Introduction
}

Resistive switches, or memristors, exhibit faster switching speeds, lower power consumption, higher scalability, and greater 3-D integration than standard complementary metaloxide-semiconductor (CMOS) circuits, thus promoting further miniaturization of next-generation neuromorphic computing components. ${ }^{1,2}$ Resistive switching is defined by a reversible change in resistance between a high resistance state (HRS) and low resistance state (LRS) that depends on 
the history of the applied external bias. ${ }^{3}$ Resistive switching phenomena occur in numerous materials, including transitional metal oxides,,${ }^{4,5}$ nitrides, ${ }^{6}$ and complex metal oxides, such as perovskites. ${ }^{7,8}$ Fabrication of most memristive systems, such as thin films, ${ }^{9,10}$ nanowires, ${ }^{11}$ nanotubes, ${ }^{12}$ and nanodots utilizes expensive ultra-high vacuum approaches that require long deposition times. Spin-coated sol-gel $\mathrm{ZnO}$ thin films ${ }^{13}$ and hydrothermally prepared $\mathrm{TiO}_{2}$ thin films ${ }^{14}$ represent two examples of inexpensive, high-yield alternative fabrication processes reported in the last decade.

More recently solution-processed zero-dimensional nanocrystals (NCs) have garnered significant research interest as constituent materials in memristive or resistive switching applications due to their physical flexibility, low power-consumption, and low fabrication cost. ${ }^{15-}$ ${ }^{17}$. Dip-pen nanolithography, ${ }^{18}$ wet stamping, ${ }^{19}$ and spin coating ${ }^{20}$ enable solution-processed multicomponent patterning, but also require complicated, time-consuming procedures to achieve basic controllability over material microstructures. Alternatively, flow coating shows promise as a dynamic, self-assembly method that patterns highly crystalline inorganic zero-dimensional nanocrystals into ordered assemblies over large areas. ${ }^{21-23}$

Memristive behavior was recently demonstrated in blade-assisted nanoribbon assemblies comprising single-crystalline $\mathrm{SrTiO}_{3}$ nanocubes capped with oleic acid ligands that were able to be transferred to a second, arbitrary surface and modulate switching direction. ${ }^{23}$ A comparison of various carboxylic acid ligands showed that shorter ligands exhibited superior operating parameters, such as lower set/reset voltage, and higher LRS/HRS ratios in $\mathrm{HfO}_{2}$ nanoribbons. ${ }^{22}$ Subsequently, the use of phosphonic acids, such as 2-ethylhexyl phosphonic acid (EHPA) produced a highly dispersed system and an ultra-thin ligand shell that reduced inter-particle 
distances and increased the tunneling probability ${ }^{24,25}$ to greatly reduce set/threshold voltages compared to solution-processed memristors comprising carboxylic acids. ${ }^{23}$

Here we use a facile flow coating method to prepare nanoribbon assemblies comprising various sizes $\mathrm{BZO}(2.3 \mathrm{~nm}$ and $4.5 \mathrm{~nm})$ and $\mathrm{SZO}(2.4 \mathrm{~nm}$ and $9 \mathrm{~nm})$ nanocrystals capped with 2hexyldecylphosphonic $\operatorname{acid}^{21}$ as a platform to study memristive phenomena in solution-processed mixtures. Reports of resistive switching behavior in BZO and SZO have thus far been restricted to vacuum deposited thin films, ${ }^{26-28}$ where the transformation from bipolar to unipolar switching was observed in a $\mathrm{SrRuO}_{3} / \mathrm{Cr}: \mathrm{SZO} / \mathrm{Pt}$ stack induced by Joule heating ${ }^{29}$ while simultaneous resistive switching and switchable diode phenomena were reported in $\mathrm{Ag} / \mathrm{BaZrO}_{3} / \mathrm{SrRuO}_{3}$ multilayers. ${ }^{30} \mathrm{In}$ this work we demonstrate the effects of nanoparticle size, polydispersity, and heterogeneity on resistive switching mechanism (bipolar/threshold) and operating voltages $\left(V_{S E T} / V_{R E S E T} / V_{T H}\right)$ by observing the local response of $\mathrm{BZO}, \mathrm{SZO}$ nanoribbon assemblies and their respective mixtures.

\section{Experimental Section}

\section{$\mathrm{BaZrO}_{3}$ and $\mathrm{SrZrO}_{3}$ Nanocrystal Synthesis}

Barium isopropoxide, strontium isopropoxide and zirconium isopropoxide (Alfa Aesar, > 98\%): potassium hydroxide (Sigma Aldrich, $\geq 85 \%$ ), ethanol (Acros Organics, 99.5\%), toluene (>99\%, Acros Organics) and 2-[2-(2-Methoxyethoxy)ethoxy]acetic acid (Sigma Aldrich, technical) were used without further purification. 2-hexyldecylphosphonic acid (HDPA) was synthetized as

previously detailed. ${ }^{21}$ Double metal oxide nanocrystals with composition $\mathrm{BaZrO}_{3}(\mathrm{BZO})$ and $\mathrm{SrZrO}_{3}(\mathrm{SZO})$ were synthetized via a microwave-assisted solvothermal method ${ }^{31}$ according to an established procedure. Custom bimetallic precursors were prepared from individual isopropoxides metal sources via alcohol thermal exchange. The resulting materials $\left(0.2 \mathrm{~g}, 0.30 \mathrm{mmol}\left(\mathrm{BaZrOR}_{6}\right)\right.$ and $\left.0.31 \mathrm{mmol}\left(\mathrm{SrZrOR}_{6}\right)\right)$ were subjected to a thermal process in a CEM Discovery SP microwave 
(max. output $300 \mathrm{~W}$ ) in a basic $\mathrm{KOH}$ ethanol solution $(4 \mathrm{~mL})$. The thermal process included a plateau for $30 \mathrm{~min}$. The main parameters (Table S1) used to optimize and obtain the desired final crystallite size (Figure S1) include the base concentration and the set temperature. Recovered nanosuspensions were washed three times with acetone and centrifuged at $5000 \mathrm{rpm}$ for $2 \mathrm{~min}$. SZO nanopowders can then be stabilized directly with 2-hexyldecylphosphonic acid in toluene. An excess amount of ligand was employed followed by ultrasonic bath to ensure a good dispersibility. The excess was then removed by successively precipitating the particles with acetone and redispersing the solid in toluene. The BZO nanocrystals were first stabilized in 2-[2(2-Methoxyethoxy) ethoxy] acetic acid) (MEEAA) with methanol as the solvent. The exchange was performed by dropwise adding the BZO solution into a 2-hexyldecylphosphonic acid solution in toluene, so that the BZO particles precipitate out. The solid was recovered by centrifugation and redispersed in toluene. Further purification was performed until no MEEAA peaks were observed in the ${ }^{1} \mathrm{H}-\mathrm{NMR}$ spectra (Bruker $300 \mathrm{MHz}$ Avance I Ultrashield).

\section{Nanoribbon Assembly via Blade-Assisted Flow Coating}

A silicon wafer (University Wafer, $\langle 100\rangle, 500 \mu \mathrm{m}$ thickness, undoped) coated with $5 \mathrm{~nm}$ Ti and $35 \mathrm{~nm}$ Pt by thermal evaporator (CHA SE-600) served as the bottom electrode. The flowcoating process was conducted in a custom-built instrument that consists of a razor blade and substrate attached to a computer-controlled nanopositioner (Burleigh Inchworm controller 8200). Approximately 3 - $5 \mu \mathrm{L}$ of the nanoparticle containing solution was injected in between the blade and the substrate and was confined due to capillary forces. For the mixed/polymodal nanoribbon assemblies, equal amounts $(100 \mu \mathrm{L})$ of nanocrystal solution were pre-combined using an ultrasonic bath before injecting the aliquot under the blade. The nanopositioner was programmed for 'stopand-go' motion, where the stopping time $\left(t_{d}\right)$, step size $(d)$, and substrate moving velocity $(v)$ 
dictated the overall nanoribbon assembly microstructure. Slow nanopositioner speeds $(1 \mathrm{~mm} / \mathrm{sec})$ and no stopping time yielded a thin film structure. The substrate moving velocity and the nanoparticle solution concentration controlled the film thickness. A pronounced, quasi-nanoribbon structure was formed at the edge of thin film due to the initial solution injection between the blade and substrate (Figure S2).

\section{Conductive Atomic Force Microscopy (C-AFM)}

The local switching response was measured under ambient conditions at room temperature using an Asylum Research Cypher ES atomic force microscope. A conductive solid Pt cantilever (Rocky Mountain Nanotech 25Pt400B; $k=8 \mathrm{~N} / \mathrm{m} ; f=10 \mathrm{kHz} ; r_{t i p}<35 \mathrm{~nm}$ ) was used to perform all topographic and conductive imaging. Conductive silver paste (Leitsilber, TED PELLA, Inc.) fixed the sample bottom electrode to the conductive wired sample puck to complete the C-AFM

electrical circuit. The conductive cantilever was connected to an Asylum Research ORCA ${ }^{\mathrm{TM}}$ dual gain transimpedance amplifier and held at ground while the sample was biased from the bottom electrode during electrical testing. Local resistive switching I-V curves were collected in currentvoltage (I-V) spectroscopy point-mode. The bias applied to Pt substrate ranged from $1 \mathrm{~V}$ to $10 \mathrm{~V}$ depending on the actual nanoribbon onset voltage at a sweep frequency of $0.99 \mathrm{~Hz}$. The cantilever deflection setpoint was held at $0.02 \mathrm{~V}$ to ensure sufficient tip-sample contact without mechanically inducing artificially high currents. State retention measurements involved first inducing the LRS, then applying a read voltage of $0.6 \mathrm{~V}$ for a nominal time of 2000 seconds. Subsequently, the bias sweep induced the HRS and the read voltage applied again.

\section{UV-Vis Absorption Measurements}

Absorption study of all nanocrystal systems was conducted using an ultraviolet-visible scanning spectrophotometer (UV/Vis, Genesys 10S, Thermo Scientific). The BZO-2.3, BZO-4.5; SZO-2.4, 
SZO-9.0 nanocrystal systems were each dispersed in $2-3 \mathrm{~mL}$ of toluene and placed in a quartz cuvette ( $3 \mathrm{~mL}, 10 \times 10 \mathrm{~mm}$ cell, Jasco Corporation); pure toluene (Fisher Scientific) was used as the reference standard. Spectrum for each nanocrystal suspension was collected across the range of $190-1100 \mathrm{~nm}$ using a $1 \mathrm{~nm}$ increment. The spectrum collection process was repeated three times for each system to ensure accuracy.

\section{Results and Discussion}

\section{Flow Coating of Nanoribbon Assemblies Comprising Perovskite Zirconate Nanocrystals}
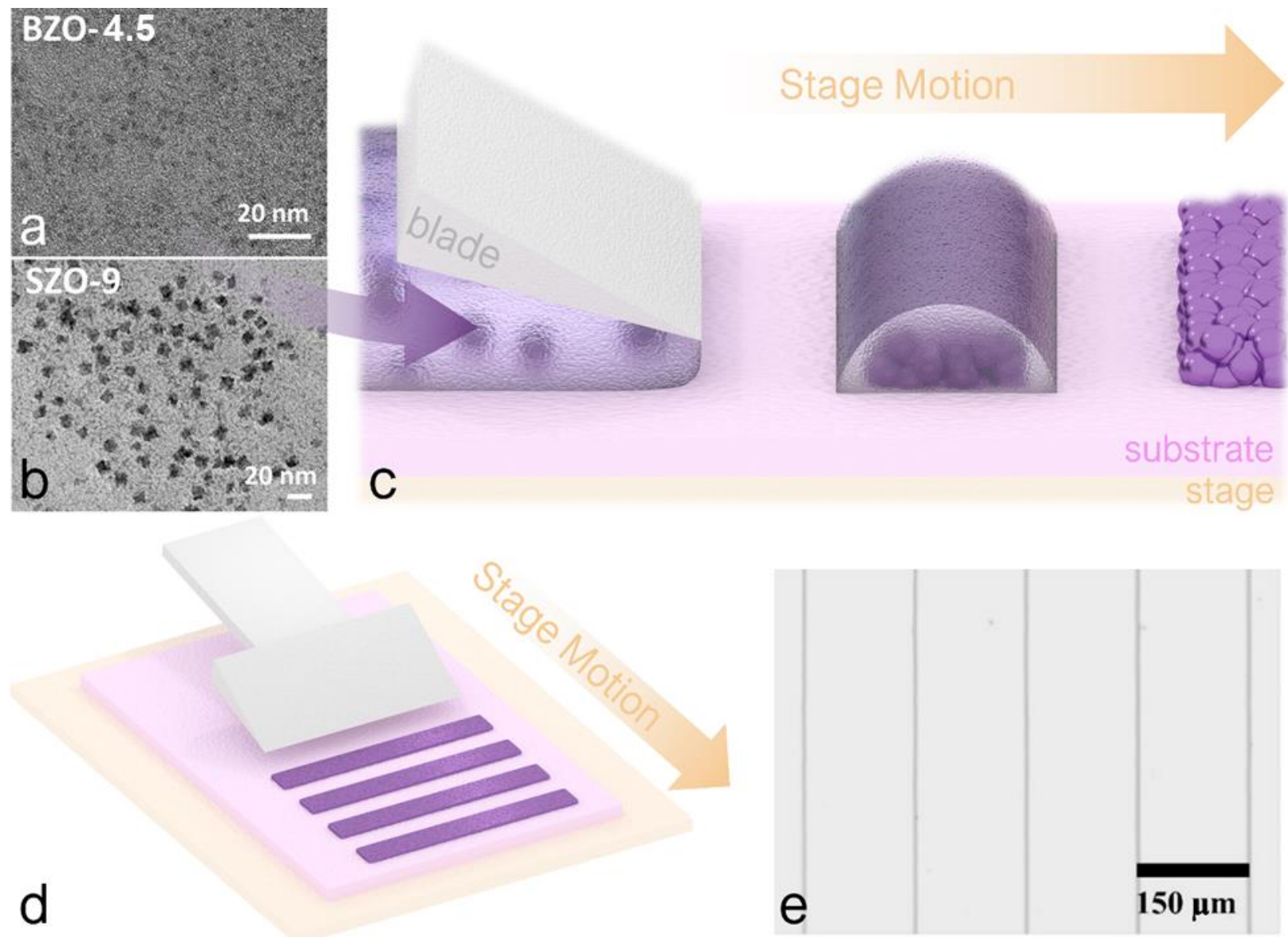

Figure 1: a,b - Low resolution TEM images of BZO-4.5 and SZO-9.0 nanocrystals, respectively. c - Nanocrystal solutions are injected under a blade against a rigid substrate, where d - "stop-and-go" motion of the stage produces nanocrystal ribbon assemblies to form, as shown in e, an optical micrograph of BZO-4.5 nanocrystal ribbon assembly arrays.

$\mathrm{BaZrO}_{3}$ and $\mathrm{SrZrO}_{3}$ nanocrystals (NCs) were synthesized via a microwave-assisted solvothermal method, ${ }^{31}$ and functionalized with 2-hexyldecylphosphonic acid (HDPA). ${ }^{21}$ Deposition of BZO and SZO nanoribbon assemblies required the implementation of flow coating 
technique, previously demonstrated for $\mathrm{HfO}_{2}^{21,22}$ and $\mathrm{SrTiO}_{3}{ }^{23}$ nanocrystal nanoassemblies. Nanoribbons comprising different diameter NCs, labelled hereafter as BZO-2.3, BZO-4.5, SZO2.4 and SZO-9 respectively (Figure S1), were deposited onto a silicon substrate coated with $5 \mathrm{~nm}$ Ti and $35 \mathrm{~nm}$ Pt as the bottom electrode.

Figure 1a and 1b are the transmission electron microscopy (TEM) images of as-prepared BZO-4.5 and SZO-9.0 NCs. X-ray diffraction confirmed the presence of reflections indexed to crystalline structure of cubic $\mathrm{BaZrO}_{3}{ }^{32}$ and $\mathrm{SrZrO}_{3}{ }^{33}$ (Figure S3). The flow coating technique, as shown in Figure 1c, deposits the nanoparticle solution between a fixed blade and a rigid substrate. Nanocrystals form ordered ribbon-like structures as volatile organic solvents evaporate in ambient air (Figure 1d), as shown by the optical micrograph of nanoribbon assembly arrays of BZO-4.5 in Figure 1e. Topographic AFM images, optical micrographs, and flow coating time versus nanoribbon dimensions can be found in Figure S2.

\section{Resistive Switching Response of Individual Nanoribbon Assemblies via C-AFM}
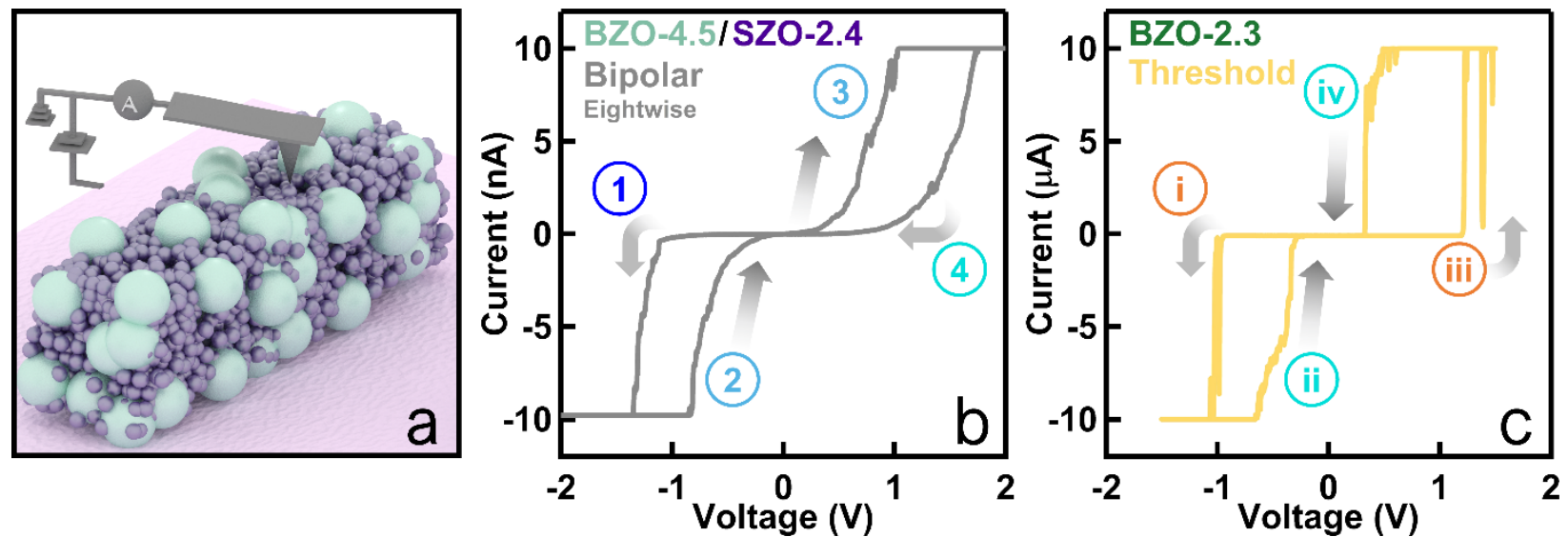

Figure 2: a, A schematic illustration of the c-AFM measurement geometry across an individual polymodal nanoribbon assembly. b, The I-V response of a BZO-4.5/SZO-2.4 polymodal nanoribbon (height $=80 \mathrm{~nm}$ ) displaying "eightwise" bipolar resistive switching character, as indicated by the arrow markers. Here " 1 " indicates the SET process, " 2 " and " 3 " the LRS response, and " 4 " the RESET process. c, The I-V response of an individual BZO-2.3 monomodal nanoribbon displaying (volatile) threshold switching behavior. Here "i" and "iii" indicates the threshold voltage and increase to the LRS; "ii" and "iv" initiate the return to the LRS.

Conductive AFM (c-AFM) represents a powerful tool used in nanoscale resistive switching studies, where a bias is applied to the substrate to probe the local charge transport and current- 
voltage (I-V) character of nanocrystal nanoribbon assemblies using the conductive tip as a (grounded) translatable top electrode (Fig. 2a). Here direct contact of a pure Pt probe with the surface of an individual nanoribbon created a $\mathrm{Pt} / \mathrm{BZO}(\mathrm{SZO}) / \mathrm{Pt}$ test structure.

Prior to electroforming with a large, applied voltage no current is observed across the middle of the nanoribbon assembly. Since the applied voltage is limited to $10 \mathrm{~V}$ due to AFM instrumentation protection, electroforming required maintaining a bias of $10 \mathrm{~V}$ for longer time periods (between 30 and 60 s). During the electroforming process, a positive voltage applied to the bottom electrode creates a high electric field that enables the creation of oxygen vacancies within the nanocrystals and induces migration toward the tip along the inter-nanocrystal "grain" boundary to form a low resistance conductive path, ${ }^{34}$ yielding a bright spot in the c-AFM current image.

\section{Coexistence of Bipolar Resistive Switching and Threshold Switching}

Figures 2b, S4, and S5 display the characteristic current-voltage (I-V) bipolar switching response of an individual nanocrystal nanoribbon assembly, as collected using a sweeping voltage (between \pm 1 and $\pm 5 \mathrm{~V}, 1 \mathrm{~Hz}$ ) applied to the Pt bottom electrode, across the nanoribbon thickness, and collected by a grounded, solid Pt tip (top electrode). A voltage sweep starting with negative polarity initially exhibits a low current level at the high resistance state (HRS) until the set voltage ("1"), which induces a significant increase in current and establishes the low resistance state (LRS). The set process produces a more gradual resistance switch than the abrupt changes typically observed for filamentary-type switching. The current cut-off (I > - $10 \mu \mathrm{A}$; I > - $10 \mathrm{nA}$ )) observed at higher applied voltages results from the built-in protection limitation of the dual-gain transimpedance amplifier in the cantilever holder (Asylum Research ORCA ${ }^{\mathrm{TM}}$ Module). The LRS remains after removal of the applied voltage, confirming the nonvolatile nature of the nanoribbons. 
As the applied bias cycles through the LRS (“2"-“3") and increases towards the maximum positive bias ( +1 to $5 \mathrm{~V}$ ) the current reduces and returns to the HRS at the observed reset voltage ("4"). Both "eightwise" (Figures 2b; S4a-c) and "counter-eightwise” (Fig. S4d) (“1”-“2".-“3”-_“4”) hysteretic, bipolar resistive switching responses were observed for $\mathrm{BZO}$ and $\mathrm{BZO} / \mathrm{SZO}$ nanocrystal nanoribbon assemblies. The BZO-2.3/SZO-9.0, BZO-4.5/SZO-2.4, and BZO4.5/SZO-9.0 mixed systems exhibited "eightwise" (counter-clockwise) switching, while the BZO4.5 system exhibited "counter-eightwise" (clockwise) switching. The coexistence of bipolar switching with opposite polarity trends similarly to studies of $\mathrm{SrTiO}_{3}{ }^{10}$ and $\mathrm{TiO}_{2}{ }^{35}$ thin films, which attributed the conversion of eightwise-to-counter-eightwise polarity to local changes in the defect density. Oxygen exchange at the Pt electrode interface facilitates variations in local vacancy concentration and the migration of oxygen vacancies, ${ }^{10}$ whose presence near the Schottky contact modulates the depletion region (tunneling width) and barrier height to dictate the observed polarity of the HRS and LRS. ${ }^{36}$ In the initial Pt/BZO(SZO)/Pt test structure, oxygen vacancies at the electrode/ribbon interface serve as trapping states. During eightwise switching an applied bias promotes oxygen exchange at the Pt/oxide interface such that trap/detrap processes modulate the barrier height across the narrow depletion width. For counter-eightwise switching enhanced oxygen vacancy migration occurs along extended defects that serve as fast migration pathways towards the cathode, resulting in a larger barrier/tunneling width for additional injected carriers.

Most of the nanoribbon assembly combinations exhibited a threshold switching response. All threshold switching currents measured by c-AFM I-V point-mode spectroscopy saturated at $10 \mu \mathrm{A}$ (SZO-2.4 at $10 \mathrm{nA}$ ), the (protected) maximum current level measured by the dual-gain transamplifier in the cantilever holder. Figures $\mathbf{2 c}$, $\mathbf{S 6}$, and $\mathbf{S 7}$ show the typical threshold response of an individual nanocrystal nanoribbon assembly. Like the bipolar response (“1"_“2”.“3”_“4”) 
observed in Fig. 2b, a minimal current is observed as the negative bias sweep begins, followed by the onset of the LRS, now defined at a threshold voltage ("i"), Fig. 2c) instead of a set voltage ("1", Fig. 2b). Unlike the nonvolatile bipolar response, where the LRS is maintained through origin ("2"-“3") towards the maximum positive bias, the volatile threshold response returns to the lowcurrent HRS at zero bias ("ii"); as the bias continues to sweep, the HRS is maintained in the opposite polarity until the threshold bias is met in the second quadrant ("iii"). As the sweep continues through point "iv", the response again returns to the HRS at zero bias.

Table 1: Average values of $V_{S E T} / V_{R E S E T}$ for bipolar switching systems (grey) and $\mathrm{V}_{\mathrm{TH}}$ of threshold switching systems (yellow) comprising various diameter BZO (green) and SZO (purple) nanocrystals and their mixtures.

\begin{tabular}{|c|c|c|c|c|c|}
\hline \multicolumn{4}{|c|}{ Bipolar Switching } & \multicolumn{2}{|c|}{ Threshold Switching } \\
\hline Material & $\begin{array}{c}\text { SET } \\
\text { Voltage (V) }\end{array}$ & $\begin{array}{c}\text { RESET Voltage } \\
\text { (V) }\end{array}$ & $\begin{array}{l}\text { Switching } \\
\text { Direction }\end{array}$ & Material & $\begin{array}{c}\text { Threshold } \\
\text { Voltage (V) }\end{array}$ \\
\hline BZO-4.5 & $1.64 \pm 0.17$ & $-1.72 \pm 0.22$ & $\begin{array}{l}\text { Counter- } \\
\text { Eightwise }\end{array}$ & $\begin{array}{l}\text { BZO-2.3 } \\
\text { SZO-2.4 }\end{array}$ & $0.90 \pm 0.32$ \\
\hline $\begin{array}{l}\text { BZO-4.5 } \\
\text { SZO-2.4 }\end{array}$ & $-1.31 \pm 0.23$ & $1.48 \pm 0.40$ & Eightwise & $\begin{array}{l}\text { BZO-2.3 } \\
\text { BZO-4.5 }\end{array}$ & $1.37 \pm 0.22$ \\
\hline $\begin{array}{l}\text { BZO-4.5 } \\
\text { SZO-9.0 }\end{array}$ & $-1.68 \pm 0.45$ & $2.85 \pm 0.40$ & Eightwise & BZO-2.3 & $1.39 \pm 0.19$ \\
\hline $\begin{array}{l}\text { BZO-2.3 } \\
\text { SZO-9.0 }\end{array}$ & $-2.19 \pm 0.34$ & $2.99 \pm 0.71$ & Eightwise & SZO-2.4 & $1.53 \pm 0.16$ \\
\hline & & & & $\begin{array}{l}\text { SZO-2.4 } \\
\text { SZO-9.0 }\end{array}$ & $1.71 \pm 0.16$ \\
\hline & & & & SZO-9.0 & $4.18 \pm 1.03$ \\
\hline
\end{tabular}

Table 1 shows the average $V_{S E T}$ and $V_{R E S E T}$ values and switching directions for the nanocrystal assemblies exhibiting bipolar switching behavior (grey), and the average $V_{T H}$ values 
for assemblies exhibiting threshold switching behavior (maize). Set/reset voltages were taken as absolute values to account for switching direction in bipolar systems. Bipolar switching systems exhibited a trend where $V_{\text {SETRESET }}$ of SZO-2.4/BZO-4.5 nm diameter polymodal assemblies < $V_{\text {SET/RESET }}$ of BZO-4.5 nm monomodal assemblies $<V_{\text {SET/RESET }}$ of polymodal assemblies partially comprising SZO-9.0 nm nanocrystals. Threshold switching systems exhibited similar trends with respect to threshold voltage: $V_{T H}$ of polymodal assemblies with small diameter nanocrystals (SZO2.4/BZO-2.3 nm; BZO-2.3/BZO-4.5 nm) $<V_{T H}$ of small diameter monomodal assemblies (SZO$2.4 \mathrm{~nm}$ or BZO-2.3 nm) $<V_{T H}$ of polymodal assemblies with large diameter nanocrystals (SZO2.4/SZO-9.0 nm) $<V_{T H}$ of large diameter monomodal assemblies (SZO-9.0 nm).

Previous studies that either changed the temperature, ${ }^{37}$ varied the electrode thickness, ${ }^{38}$ controlled the current compliance, ${ }^{39}$ or tuned the oxygen stoichiometry ${ }^{40-43}$ all produced instabilities in oxide thin films that resulted in the conversion of bipolar resistive switching to threshold switching. All measurements of the nanoribbon assemblies were performed at ambient temperature, so environmental temperature is not initiating the bipolar-to-threshold transition. In filamentary-based switching, competing Joule heating and thermal dissipation processes results in instability of the low resistance state and can results in threshold switching. ${ }^{37}$ While I-V fitting indicates the nanoribbon systems do not exhibit filamentary-based switching, the $40 \mathrm{~nm}$ electrodes $(5 \mathrm{~nm} \mathrm{Ti} / 35 \mathrm{~nm} \mathrm{Pt})$ coincidentally fall on the same critical bottom electrode thickness between threshold and memory switching observed for $\mathrm{NiO}$ systems. ${ }^{38}$ It is likely that inefficient heat dissipation caused by thinner electrodes partially contributes to observance of either bipolar or threshold switching for the family of BZO/SZO nanoribbon assemblies here. Studies utilizing current compliance control leverage high values $\left(\mathrm{I}_{\mathrm{CC}}>100 \mu \mathrm{A}\right)$ during the set process to induce structural changes that stabilize the formed filament. ${ }^{39-41}$ As most c-AFM amplifiers do not use a 
current compliance, and the instrument protection limits the current to only $10 \mu \mathrm{A}$, it is possible that the electric field is insufficient to migrate enough vacancies to induce bipolar switching.

\section{Size-Dependent Oxygen Vacancy Modulation}

Figure 3 (a) shows the cumulative probability of SET voltage in BZO and SZO monomodal nanocrystal assemblies. In both cases the smaller diameter monomodal nanocrystal assemblies exhibited lower SET voltages and more uniform switching behavior, indicated by the lower coefficient of variation (CV) values. The SZO-9.0 system exhibited anomalously high average SET voltages across a broad range from $1.64 \mathrm{~V}$ to $5.98 \mathrm{~V}$. Figure 3 (b) shows the threshold switching cycling response of an individual BZO-2.3 nanocrystal nanoribbon assembly at the read voltage of $1.25 \mathrm{~V}$. A selectivity of $\sim 10^{2}-10^{3}$, even without current compliance, provides evidence of stable switching in the system. State retention tests of the BZO-2.3 (Fig. 3c), SZO-2.4 (Fig. 3d), and mixed BZO-2.3/SZO-2.4 (Fig. S8) nanoassemblies all demonstrated stability for 2000 seconds.

Contributing factors to the variations in operating voltage and uniformity (stability) likely include a strong influence from size-dependent nanocrystal structural changes. A comprehensive survey of XRD, TEM, and DFT studies concluded that size-dependent lattice expansion commonly occurs for binary and complex oxide nanocrystal systems, due to multiple factors involving modulation of the surface stress based on variations of the surface-to-volume ratio and the resulting interactions with the surrounding environment (adsorbates). ${ }^{44}$ Thus smaller BZO and SZO nanocrystals likely possess higher lattice parameters than their larger diameter nanocrystal counterparts. The larger unit cell experiences more structural fluctuations and subsequently increases oxygen vacancy diffusion. ${ }^{45}$ The point defects (oxygen vacancies) also actively facilitate the resistive switching process and thus lower the switching voltage. ${ }^{46}$ 
The Tauc method was applied to collective UV-vis absorption spectra (Figure S9) to estimate the band gaps for BZO (Fig. S10a) and SZO (Fig. S10b) nanocrystal systems. The band gaps of both the BZO and SZO systems displayed a distinct red-shift with decreasing nanocrystal diameter. The narrowing band gap energy is attributed to an increase in oxygen vacancy concentration in smaller diameter nanocrystals, which ultimately raises the valence band maximum. ${ }^{47}$ In these zirconate systems the valence band comprises $\mathrm{O} 2 p$ states while the conduction band mainly comprises $\mathrm{Zr} 4 d\left(\mathrm{t}_{2 \mathrm{~g}}\right)$ states. $^{48}$ The increased presence of oxygen vacancies create localized intra-band gap defect states (e.g. F-centers, $\mathrm{F}^{0}$-centers ${ }^{49}$ that significantly alter the $p$ - $d$ charge transfer transitions and subsequently reduce the band gap. ${ }^{50}$ The increased vacancy concentration manifests as both lower SET (threshold) voltage and improved switching uniformity for nanocrystal assemblies, similar to recent studies of $\mathrm{LaAlO}_{3},{ }^{51} \mathrm{LaMnO}_{3},{ }^{52}$ and $\mathrm{BiFeO}_{3} .{ }^{53}$

Figure 3: a, A plo
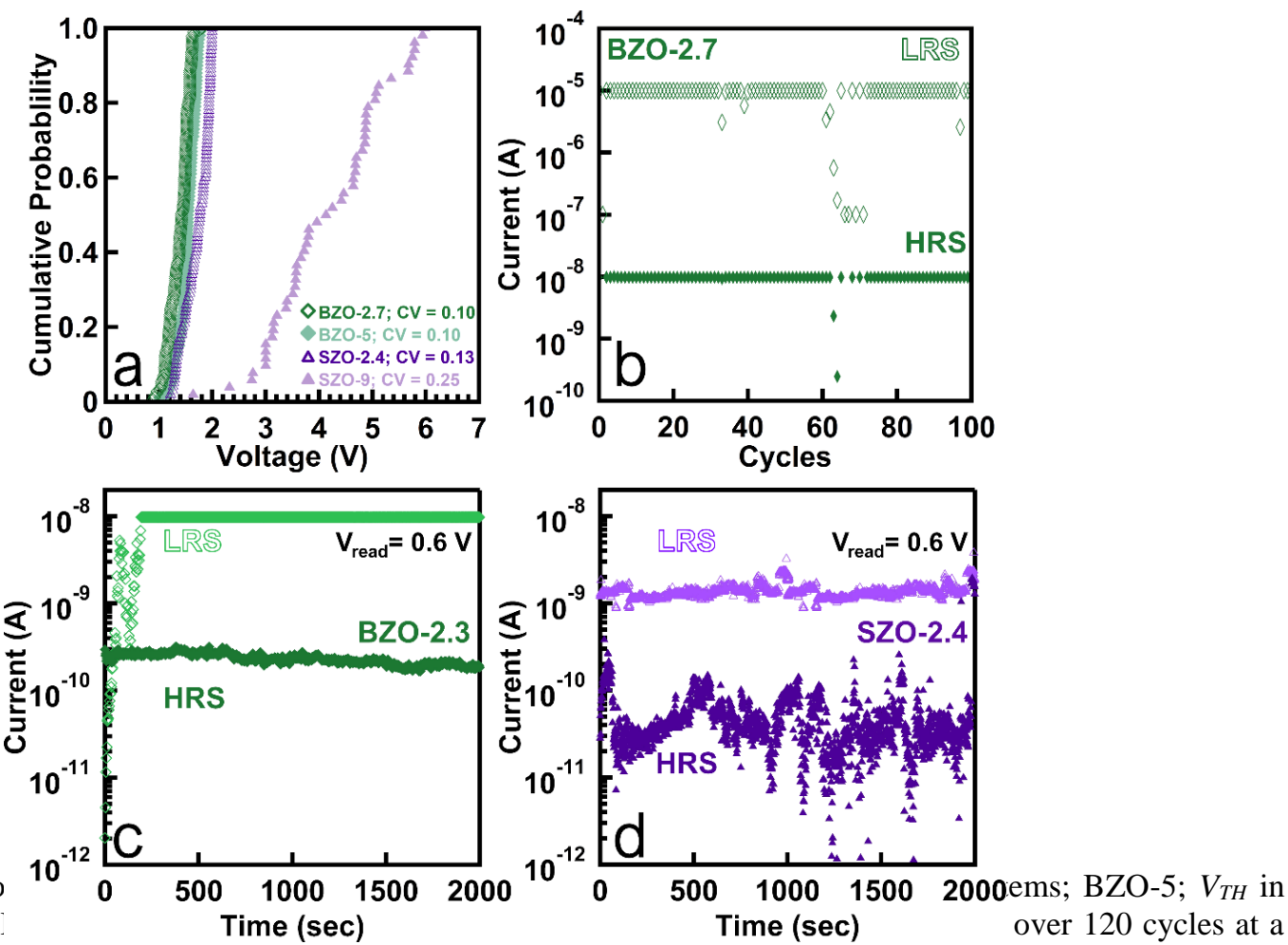
read voltage of $1.25 \mathrm{~V}$ and displaying a selectivity of $\geq 10^{3}$. LRS and HRS state retention tests for the $\mathbf{c}$, BZO-2.3 and d, SZO 2.4 nanoassemblies.

\section{Trap-Mediated Space Charge Limited Current}


The current-voltage responses of the 10 BZO-SZO combinations (Figs 2b, 2c, S4, S6) were plotted on a log-log scale and fitted to determine the governing switching mechanism(s). Electron traps may occur in some combination of inter-crystal phosphonic acid (PA) ligands and positivelycharged oxygen vacancies accumulated at nanocrystal interfaces (and fixed at the tip-nanocrystal interface). The underlying mechanism driving the observed current of ligand-capped nanocrystal assemblies depends strongly on ligand length. ${ }^{25,54}$ Longer organic ligands result in larger intercrystal distances and results in charge transport limited current. Dielectric constants for phosphonic acid ligands have been shown to be lower than that of transition metal oxides, ${ }^{55}$ thus the electric field is assumed to be primarily applied across the inter-crystal gap (ligand). ${ }^{56}$ The large, internanocrystal separation distances create intra-band trap states that drive space-charge-limited conduction, commonly observed for semiconductor quantum dot systems (Figure S11). ${ }^{25}$

Conversely, shorter ligands such as HDPA reduce the inter-crystal spacing to yield higher hopping rates, resulting in a system that is charge injection limited. Figures $4 \mathbf{a}, \mathbf{b}$ illustrate the mechanism driving the switching phenomena, like that observed for tip-induced switching in oxide nanocrystals ${ }^{23,57}$ and found within quantum dot optoelectronics. ${ }^{54}$ Bias is applied to the bottom Pt thin film electrode while the Pt-tip serves as a grounded, translatable top electrode. Recent electronic adsorption measurements showed the tendency for vacancies to populate along nanocrystal surfaces. ${ }^{50}$ A space-charge region forms within the oxygen-deficient surface layer (darker teal outer sphere, Figs. 4a,b) and impedes oxygen diffusion. ${ }^{58}$

During the initial bias sweep electrons are injected into the BZO (SZO) nanocrystal assemblies and fills the traps at the Pt tip - nanocrystal interface. Electrons are released from the 

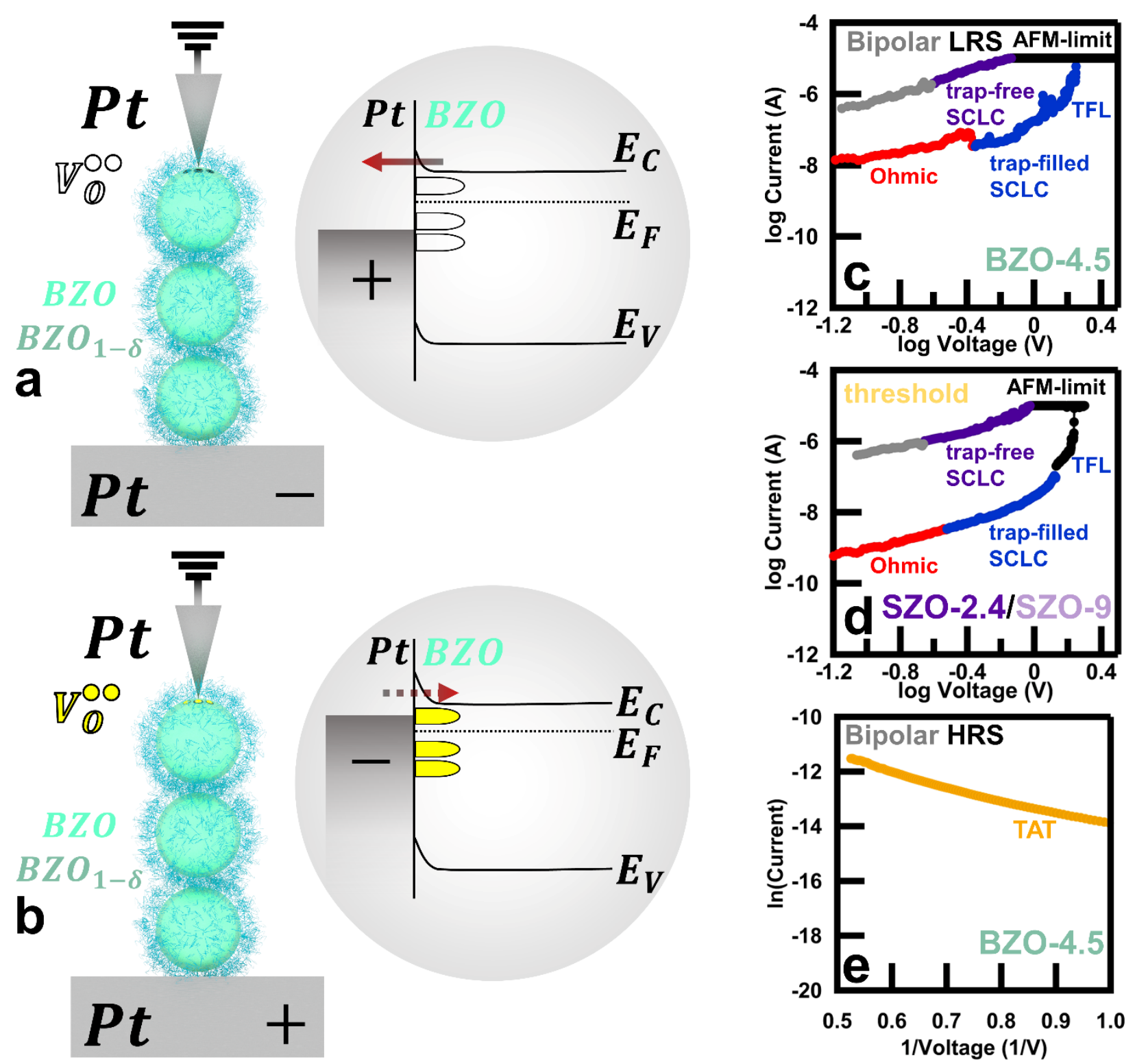

Figure 4: (a,b) An illustration of the operating switching mechanism and band structure across the $\mathrm{Pt}(\mathrm{tip}) / \mathrm{BZO}(\mathrm{SZO})$ interface. (a) An applied negative bias injects electrons from the traps (oxygen vacancies or ligands; hollow black spheres) to the tip electrode to leave unfilled traps that narrow the Schottky barrier and induce the HRS $\rightarrow$ LRS transition. (b) An applied positive bias fills traps at the interface with electrons (yellow dots in hollow black spheres) and recovers the Schottky-like barrier, initializing the LRS $\rightarrow$ HRS transition. Log-log curve fitting of (c), bipolar switching (BZO-4.5) and (d), threshold switching (SZO-2.4/SZO-9) systems exhibiting space-charge-limitedconduction (SCLC). The response exhibits four characteristic regimes: i) low-voltage ohmic conduction (slope $\mathrm{m}=1$, red); ii) trap-filled SCLC $(\mathrm{m}=2$, blue); iii) trap-filled limit $(\mathrm{m}>2)$; iv) trap-free SCLC (m =2, purple). The AFMlimit indicates the $10 \mu \mathrm{A}$ instrument protection on the current amplifier. (e) ln-I vs 1/V plot displaying the linear trend associated with trap-assisted tunneling (TAT) for the HRS of bipolar resistive switching nanoassemblies (BZO-4.5).

traps at larger negative voltages (applied at the bottom electrode) to yield unfilled traps, reduce the Schottky barrier width, and enable the HRS $\rightarrow$ LRS transition (Fig. 2b; “1”; Fig. 4a). Large positive voltages applied to the bottom electrode refills these interfacial traps with electrons to 
maintain neutrality with the positively-charged oxygen vacancies, thus increasing the Schottky barrier width and initializing the LRS $\rightarrow$ HRS transition (Fig. 2b; "4"; Fig. 4b). Therefore, charge trap/detrap cycles at the tip-sample interface facilitated by interfacial oxygen vacancy electron traps $^{59-61}$ define the conduction mechanism for the observed c-AFM current response of the nanocrystal ribbon assemblies. The log-log fitting shown in Figure 4 indicates that the LRS in the bipolar (Fig. 4c) and threshold switching (Fig. 4d) systems exhibit the four distinct regimes of space-charge-limited-conduction (current) $(\mathrm{SCLC}):^{62,63}$ i) ohmic-like conduction $(m=1, I \propto$ $V^{m}$, red $)$; ii) trap-limited space-charge-limited-current $(S C L C, m=2$, blue $)$; iii) the trap-filled limit $\left(V_{T F L}, m>2\right)$; iv) trap-free SCLC (Child's Law; $\left.m=2\right)$. The HRS response (Fig. 4e) trends linearly with a fit of the trap-assisted tunneling model (TAT) ${ }^{63}$ confirming the trapmediated process at the Pt/nanocrystal interface. All other log-log plots with fitting can be found in the Supporting Information, Figures S12 and S13.

\section{Packing Density Considerations}

The packing efficiency of multi-diameter constituent nanocrystals also likely contributes in part to overall transport and variations in the switching voltages. A higher nanocrystal packing density is known to reduce inter-particle distance and less inter-particle carrier hopping per unit length for

transport across the assembly. ${ }^{25}$ Spherical systems exhibiting bimodal size distributions tend to produce higher packing factors than monomodal size distributions. ${ }^{64}$ Since the C-AFM measurements were conducted at an $80 \mathrm{~nm}$ equivalent thickness area with a tip radius of $35 \mathrm{~nm}$, the electrical field was assumed to be confined to a cylindrical container with $\mathrm{r}=40 \mathrm{~nm}$ and $\mathrm{h}=$ $80 \mathrm{~nm}$ (Figure 5a). The packing factor was then calculated for both BZO and SZO using the expressions for monomodal and bimodal spheres in a cylindrical container (see Supporting Info). ${ }^{65}$ 

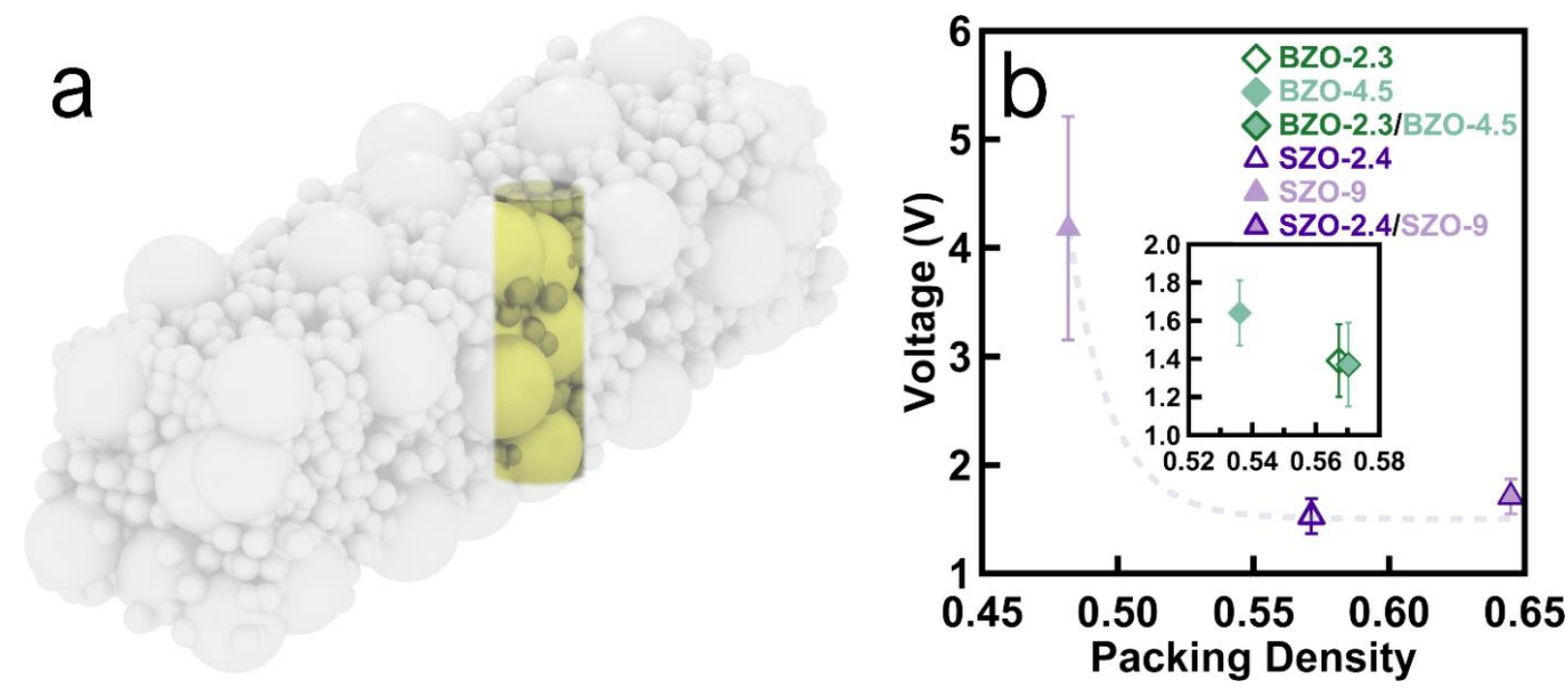

Figure 5: a, An illustration of the cylindrical container directly under the tip used in packing calculations. b, A plot of onset (threshold) voltage for SZO constituent nanocrystals and their mixtures versus calculated packing density for monomodal and bimodal assemblies. The dotted double exponential fit serves as a guide for the eye. Inset: the $V_{S E T}$ (or $V_{T H}$ ) for BZO constituent nanocrystals and their mixtures versus packing density, displaying a linear trend.

Figure 5b shows the onset threshold voltage versus calculated packing density for both monomodal and bimodal SZO nanoribbon assemblies, where the inset shows the resistive switching SET/threshold voltage for BZO nanoassemblies. For SZO systems the onset voltage decreases with increasing packing density, nearly following a double exponential fit; BZO systems decrease with packing density linearly across a small range around the SZO-2.4 data point. Studies of PbSe nanocrystal field effect transistors demonstrated a relatively insensitivity of mobilities and transport to polydispersity, as some population of larger nanocrystals provided a percolative network. $^{25}$ While it is possible the assemblies did not meet the percolation threshold, the homogenous mixtures (BZO 2.3/4.5; SZO 2.4/9) exhibited switching voltages similar to the smaller nanocrystal systems. This suggests that the increase in vacancy concentration for smaller nanocrystal provides additional interfacial surface traps that dictate the charge-injection-limited transport described in Figure 4. Packing density is one of many variables that partially contribute to the switching response. Changes in nanocrystal size also affect its shape, surface charge distribution, and surface trap density, ${ }^{25,66}$ while the "free" volume comprises both ligand-filled and 
void space. These results suggest a fully comprehensive mechanistic understanding of solutionprocessed memristors will require a series of studies to decouple the strong interplay between size, composition, and defect chemistry and its effects on switching.

\section{Zirconates: A-site Substitutional Considerations}

In considering composition vs defect chemistry, we note that the BZO system exhibits a lower operating voltage than the SZO system for nearly identical nanocrystal diameters, suggesting that compositional contributions couple strongly with the size-dependent response. BZO-2.3 displayed an average threshold voltage of $1.39 \pm 0.19 \mathrm{~V}$ while SZO-2.4 displayed an average threshold voltage of $1.53 \pm 0.16 \mathrm{~V}$, resulting in a significant statistical difference with a calculated p-value less than 0.0001. Recent studies of these $\mathrm{ABO}_{3}$ perovskite-type zirconates show that $\mathrm{BZO}$ nanoparticles possess significantly more A-site vacancies than SZO nanoparticles because the slower Ba diffusivity (500 times slower than $\mathrm{Sr}$ in $\mathrm{SZO}$ ) during synthesis results in fewer A-site vacancies being filled and more oxygen vacancies introduced due to charge compensation. ${ }^{67,68}$ Other DFT studies showed that $V_{O}^{\bullet}$ represent the primary point defects for $\mathrm{BaZrO}_{3}$ while the comparatively smaller cation ionic radii of $\mathrm{Sr}$ results in preferentially-formed $\mathrm{Zr}_{S r}^{2+}$ antisites that suppress vacancy formation in $\mathrm{SrZO}_{3}$, especially in $\mathrm{Zr}$-rich stoichiometries. ${ }^{69}$

More recent DFT studies of rock-salt alkaline earths demonstrated that under an applied electric field the polarization work lowered the field-dependent formation energy of vacancies, which were shown to possess highly polarizable trapped electrons that reduced bond stiffness and became more polarizable under an applied electric field. ${ }^{70} \mathrm{We}$ posit that the higher electron donor power and dynamic bond polarizability of Ba-O facilitates vacancy formation and oxygen migration, and thus reducing the operating voltages in the observed switching response of BZO systems compared to SZO. This mechanism is further supported by the tendency for all BZO-SZO 
bimodal heterogeneous mixtures to exhibit lower voltages than either monomodal or bimodal SZO assemblies regardless of diameter.

\section{Summary}

In summary, we demonstrated the impact of nanocrystal diameter and dispersity/packing on resistive switching parameters across a library of monomodal and homogeneous bimodal $\mathrm{BaZrO}_{3}(\mathrm{BZO})$ and $\mathrm{SrZrO}_{3}(\mathrm{SZO})$ nanocrystal nanoribbon assemblies. The variations in constituent nanocrystal diameters contribute to changes in oxygen vacancy concentration and inter-gap trap states, which mediate space-charge-limited conduction and trap-assisted tunneling transport mechanisms along the tip-sample interface. The oxide exhibited respectable selectivity, stability, and state retention. Substituting the A-site cation in these zirconate perovskites impacted the switching response, as evidenced by the observation of a lower BZO $V_{S E T}$ than SZO $V_{S E T}$ for near-equivalent diameters, and lower operating voltages in heterogeneous bimodal BZO/SZO mixed nanoassemblies compared to homogeneous monomodal and bimodal SZO nanoassemblies. Moreover, Solution processing techniques such as flow coating provide a versatile, facile fabrication route for creating ordered nanoassemblies comprising complex functional nanocrystal with controllable constituent nanocrystal size, inter-particle distance, and material structure. We expect this study will motivate further investigation of size-dependent and substitutional effects of constituent nanocrystals in solution-processed and colloidal assemblies used in flexible electronics and energy applications. The switching differences observed in these multi-functional zirconate perovskites also translate to a broader family of materials with coupled and strongly-correlated phenomena such as ferroic and optoelectronic systems.

\section{Associated Content}




\section{Supporting Information:}

The Supporting Information is available free of charge on the ACS Publications website at DOI: 10.1021/acsami.XXXXXX.

Additional experimental and analysis details include nanocrystal synthesis experimental conditions and process; size distributions; x-ray diffraction patterns; nanoribbon assembly topographic imaging; plot of nanoribbon dimensions versus stop time; bipolar switching I-V curves; threshold switching I-V curves; UV-Vis absorption spectra; Tauc band-gap estimation; LRS/HRS state retention; charge transport-limited band diagram; bipolar switching log-log curve fitting; threshold switching log-log curve fitting; multimodal sphere packing calculations (PDF).

\section{Author Information}

Corresponding Author

Stephen S. Nonnenmann - Department of Mechanical and Industrial Engineering, University of Massachusetts-Amherst, Amherst, Massachusetts 01003, United States. orcid.org/0000-0002-5369-9279; Email: ssn@umass.edu.

Authors

Zimu Zhou - Department of Mechanical and Industrial Engineering, University of MassachusettsAmherst, Amherst, Massachusetts 01003, United States. orcid.org/0000-0003-3424-0566

Pedro L. Dominguez - Department of Chemistry, Ghent University, Ghent, Belgium. orcid.org/00000002-8222-6200

Muhammad Abdullah - Department of Chemistry, University of Massachusetts-Amherst, Amherst, Massachusetts 01003, United States. https://orcid.org/0000-0003-4042-4206

Dylan M. Barber - Department of Polymer Science and Engineering, University of MassachusettsAmherst, Amherst, Massachusetts 01003, United States.

Xiangxi Meng - Department of Chemical Engineering, University of Massachusetts-Amherst, Amherst, Massachusetts 01003, United States. orcid.org/0000-0003-3886-7892

Jieun Park - Department of Mechanical and Industrial Engineering, University of MassachusettsAmherst, Amherst, Massachusetts 01003, United States. orcid.org/ 0000-0003-2720-9544

Isabel Van Driessche - Department of Chemistry, Ghent University, Ghent, Belgium. https://orcid.org/0000-0001-5253-3325 
Jessica D. Schiffman - Department of Chemical Engineering, University of Massachusetts-Amherst, Amherst, Massachusetts 01003, United States. orcid.org/0000-0002-1265-5392

Alfred J. Crosby - Department of Polymer Science and Engineering, University of MassachusettsAmherst, Amherst, Massachusetts 01003, United States. orcid.org/0000-0001-8850-8869

Kevin R. Kittilstved - Department of Chemistry, University of Massachusetts-Amherst, Amherst, Massachusetts 01003, United States. orcid.org/0000-0002-9852-7454

\section{Notes}

The authors declare no competing financial interest.

\section{Acknowledgements}

Z.Z. and S.S.N. acknowledge partial support of this research through the NSF Directorate of Engineering under award CBET-1706113. P.L.D. and I.V.D acknowledge the funding received from the European Union's Horizon 2020 Research and Innovation Programme under the Marie Skłodowska-Curie Grant agreement No. 722071 (SynFoNY, www.synfony.eu). D.M.B. acknowledges a National Defense Science and Engineering Graduate (NDSEG) Fellowship. X.M. acknowledges the support of the National Science Foundation NRT-SMLS program (DGE-1545399) Z.Z. performed all nanoribbon assembly fabrication, cAFM measurements, transport analysis, and cowrote the manuscript. J.P. performed all state retention measurements. P.L.D. and I.V.D. synthesized the constituent nanocrystals. X.M., J.D.S., M.A., K.R.K. led absorption measurements and analysis. D.M.B. and A.J.C. assisted with the nanoribbon fabrication process and film formation. S.S.N. led the overall project scope, supervised general fabrication, local probe, and data analysis studies, and wrote the manuscript. The authors also thank Prof. Jonathan De Roo for useful insights and discussions.

\section{References}

(1) Waser, R.; Aono, M. Nanoionics-Based Resistive Switching Memories. Nat. Mater. 2007, 6 (11), 833-840. https://doi.org/10.1038/nmat2023.

(2) Sawa, A. Resistive Switching in Transition Metal Oxides. Materials Today. Elsevier Ltd 2008, pp 28-36. https://doi.org/10.1016/S1369-7021(08)70119-6.

(3) Yang, J. J.; Pickett, M. D.; Li, X.; Ohlberg, D. A. A.; Stewart, D. R.; Williams, R. S. Memristive Switching Mechanism for Metal/Oxide/Metal Nanodevices. Nat. Nanotechnol. 2008, 3 (7), 429433. https://doi.org/10.1038/nnano.2008.160.

(4) Lin, K. L.; Hou, T. H.; Shieh, J.; Lin, J. H.; Chou, C. T.; Lee, Y. J. Electrode Dependence of Filament Formation in $\mathrm{HfO}_{2}$ Resistive-Switching Memory. J. Appl. Phys. 2011, 109 (8). https://doi.org/10.1063/1.3567915.

(5) Kim, K. M.; Kim, G. H.; Song, S. J.; Seok, J. Y.; Lee, M. H.; Yoon, J. H.; Hwang, C. S. Electrically Configurable Electroforming and Bipolar Resistive Switching in $\mathrm{Pt} / \mathrm{TiO}_{2} / \mathrm{Pt}$ Structures . Nanotechnology 2010, 21 (30), 305203. https://doi.org/10.1088/09574484/21/30/305203.

(6) Kim, H. D.; An, H. M.; Lee, E. B.; Kim, T. G. Stable Bipolar Resistive Switching Characteristics and Resistive Switching Mechanisms Observed in Aluminum Nitride-Based ReRAM Devices. IEEE Trans. Electron Devices 2011, 58 (10), 3566-3573. 
https://doi.org/10.1109/TED.2011.2162518.

(7) Eom, C.-B.; Wu, D.; Gruverman, A.; Lu, H.; Folkman, C. M.; Zhuravlev, M. Y.; Tsymbal, E. Y.; Jang, H. W.; Felker, D.; Wang, Y.; et al. Tunneling Electroresistance Effect in Ferroelectric Tunnel Junctions at the Nanoscale. Nano Lett. 2009, 9 (10), 3539-3543. https://doi.org/10.1021/n1901754t.

(8) Wang, C.; Jin, K. J.; Xu, Z. T.; Wang, L.; Ge, C.; Lu, H. Bin; Guo, H. Z.; He, M.; Yang, G. Z. Switchable Diode Effect and Ferroelectric Resistive Switching in Epitaxial $\mathrm{BiFeO}_{3}$ Thin Films. Appl. Phys. Lett. 2011, 98 (19). https://doi.org/10.1063/1.3589814.

(9) Choi, B. J.; Jeong, D. S.; Kim, S. K.; Rohde, C.; Choi, S.; Oh, J. H.; Kim, H. J.; Hwang, C. S.; Szot, K.; Waser, R.; et al. Resistive Switching Mechanism of $\mathrm{TiO}_{2}$ Thin Films Grown by AtomicLayer Deposition. J. Appl. Phys. 2005, 98 (3). https://doi.org/10.1063/1.2001146.

(10) Muenstermann, R.; Menke, T.; Dittmann, R.; Waser, R. Coexistence of Filamentary and Homogeneous Resistive Switching in Fe-Doped $\mathrm{SrTiO}_{3}$ Thin-Film Memristive Devices. Adv. Mater. 2010, 22 (43), 4819-4822. https://doi.org/10.1002/adma.201001872.

(11) Nagashima, K.; Yanagida, T.; Oka, K.; Taniguchi, M.; Kawai, T.; Kim, J. S.; Park, B. H. Resistive Switching Multistate Nonvolatile Memory Effects in a Single Cobalt Oxide Nanowire. Nano Lett. 2010, 10 (4), 1359-1363. https://doi.org/10.1021/n19042906.

(12) Nonnenmann, S. S.; Gallo, E. M.; Spanier, J. E. Redox-Based Resistive Switching in Ferroelectric Perovskite Nanotubes. Appl. Phys. Lett. 2010, 97 (10), 1-3. https://doi.org/10.1063/1.3486224.

(13) Kim, S.; Moon, H.; Gupta, D.; Yoo, S.; Choi, Y. K. Resistive Switching Characteristics of Sol-Gel Zinc Oxide Films for Flexible Memory Applications. IEEE Trans. Electron Devices 2009, 56 (4), 696-699. https://doi.org/10.1109/TED.2009.2012522.

(14) Dongale, T. D.; Shinde, S. S.; Kamat, R. K.; Rajpure, K. Y. Nanostructured $\mathrm{TiO}_{2}$ Thin Film Memristor Using Hydrothermal Process. J. Alloys Compd. 2014, 593, 267-270. https://doi.org/10.1016/j.jallcom.2014.01.093.

(15) Guan, W.; Long, S.; Jia, R.; Liu, M. Nonvolatile Resistive Switching Memory Utilizing Gold Nanocrystals Embedded in Zirconium Oxide. Appl. Phys. Lett. 2007, 91 (6). https://doi.org/10.1063/1.2760156.

(16) Chang, W. Y.; Cheng, K. J.; Tsai, J. M.; Chen, H. J.; Chen, F.; Tsai, M. J.; Wu, T. B. Improvement of Resistive Switching Characteristics in $\mathrm{TiO}_{2}$ Thin Films with Embedded $\mathrm{Pt}$ Nanocrystals. Appl. Phys. Lett. 2009, 95 (4). https://doi.org/10.1063/1.3193656.

(17) Chen, L.; Gou, H. Y.; Sun, Q. Q.; Zhou, P.; Lu, H. L.; Wang, P. F.; Ding, S. J.; Zhang, D. Enhancement of Resistive Switching Characteristics in $\mathrm{Al}_{2} \mathrm{O}_{3}$-Based RRAM with Embedded Ruthenium Nanocrystals. IEEE Electron Device Lett. 2011, 32 (6), 794-796. https://doi.org/10.1109/LED.2011.2125774.

(18) Piner, R. D.; Zhu, J.; Xu, F.; Hong, S.; Mirkin, C. A. "Dip-Pen" Nanolithography. Science (80-. ). 1999, 283 (5402), 661-663. https://doi.org/10.1126/science.283.5402.661.

(19) Klajn, R.; Fialkowski, M.; Bensemann, I. T.; Bitner, A.; Campbell, C. J.; Bishop, K.; Smoukov, S.; Grzybowski, B. A. Multicolour Micropatterning of Thin Films of Dry Gels. Nat. Mater. 2004, 3 (10), 729-735. https://doi.org/10.1038/nmat1231.

(20) Hall, D. B.; Underhill, P.; Torkelson, J. M. Spin Coating of Thin and Ultrathin Polymer Films. Polym. Eng. Sci. 1998, 38 (12), 2039-2045. https://doi.org/10.1002/pen.10373. 
(21) De Roo, J.; Zhou, Z.; Wang, J.; Deblock, L.; Crosby, A. J.; Owen, J. S.; Nonnenmann, S. S. Synthesis of Phosphonic Acid Ligands for Nanocrystal Surface Functionalization and Solution Processed Memristors. Chem. Mater. 2018, 30 (21), 8034-8039. https://doi.org/10.1021/acs.chemmater.8b03768.

(22) Wang, J.; Choudhary, S.; De Roo, J.; De Keukeleere, K.; Van Driessche, I.; Crosby, A. J.; Nonnenmann, S. S. How Ligands Affect Resistive Switching in Solution-Processed $\mathrm{HfO}_{2}$ Nanoparticle Assemblies. ACS Appl. Mater. Interfaces 2018, acsami.7b17376. https://doi.org/10.1021/acsami.7b17376.

(23) Wang, J.; Choudhary, S.; Harrigan, W. L.; Crosby, A. J.; Kittilstved, K. R.; Nonnenmann, S. S. Transferable Memristive Nanoribbons Comprising Solution-Processed Strontium Titanate Nanocubes. ACS Appl. Mater. Interfaces 2017, 9 (12), 10847-10854. https://doi.org/10.1021/acsami.7b00220.

(24) Gilson, M. K.; Given, J. A.; Bush, B. L.; McCammon, J. A. The Statistical-Thermodynamic Basis for Computation of Binding Affinities: A Critical Review. Biophys. J. 1997, 72 (3), 1047-1069. https://doi.org/10.1016/S0006-3495(97)78756-3.

(25) Liu, Y.; Gibbs, M.; Puthussery, J.; Gaik, S.; Ihly, R.; Hillhouse, H. W.; Law, M. Dependence of Carrier Mobility on Nanocrystal Size and Ligand Length in Pbse Nanocrystal Solids. Nano Lett. 2010, 10 (5), 1960-1969. https://doi.org/10.1021/nl101284k.

(26) Lin, M. H.; Wu, M. C.; Huang, C. Y.; Lin, C. H.; Tseng, T. Y. High-Speed and Localized Resistive Switching Characteristics of Double-Layer $\mathrm{SrZrO}_{3}$ Memory Devices. J. Phys. D. Appl. Phys. 2010, 43 (29). https://doi.org/10.1088/0022-3727/43/29/295404.

(27) Guo, Z.; Zhu, L.; Zhou, J.; Sun, Z. Design Principles of Tuning Oxygen Vacancy Diffusion in $\mathrm{SrZrO}_{3}$ for Resistance Random Access Memory. J. Mater. Chem. C 2015, 3 (16), 4081-4085. https://doi.org/10.1039/c5tc00302d.

(28) Beck, A.; Bednorz, J. G.; Gerber, C.; Rossel, C.; Widmer, D. Reproducible Switching Effect in Thin Oxide Films for Memory Applications. Appl. Phys. Lett. 2000, 77 (1), 139-141. https://doi.org/10.1063/1.126902.

(29) Jo, Y.; Jung, K.; Kim, J.; Woo, H.; Han, J.; Kim, H.; Hong, J.; Lee, J. K.; Im, H. Resistance Switching Mode Transformation in $\mathrm{SrRuO}_{3} / \mathrm{Cr}$-Doped $\mathrm{SrZrO}_{3} / \mathrm{Pt}$ Frameworks via a Thermally Activated Ti out-Diffusion Process. Sci. Rep. 2014, 4, 1-7. https://doi.org/10.1038/srep07354.

(30) Ding, Y.; Xu, X.; Bhalla, A.; Yang, X.; Chen, J.; Chen, C. Switchable Diode Effect in $\mathrm{BaZrO}_{3}$ Thin Films. RSC Adv. 2016, 6 (65), 60074-60079. https://doi.org/10.1039/c6ra05668g.

(31) Díez-Sierra, J.; López-Domínguez, P.; Rijckaert, H.; Rikel, M.; Hänisch, J.; Khan, M. Z.; Falter, M.; Bennewitz, J.; Huhtinen, H.; Schäfer, S.; Müller, R.; Schunk, S. A.; Paturi, P.; Bäcker, M.; De Buysser, K.; Van Driessche, I., High Critical Current Density and Enhanced Pinning in Superconducting Films of $\mathrm{YBa}_{2} \mathrm{Cu}_{3} \mathrm{O}_{7-\delta}$ Nanocomposites with Embedded $\mathrm{BaZrO}_{3}, \mathrm{BaHfO}_{3}$, $\mathrm{BaTiO}_{3}$, and $\mathrm{SrZrO}_{3}$ Nanocrystals. ACS Appl. Nano Mater. 2020, 3 (6), 5542-5553.

(32) Kumar, H. P.; Vijayakumar, C.; George, C. N.; Solomon, S.; Jose, R.; Thomas, J. K.; Koshy, J. Characterization and Sintering of $\mathrm{BaZrO}_{3}$ Nanoparticles Synthesized through a Single-Step Combustion Process. J. Alloys Compd. 2008, 458 (1-2), 528-531. https://doi.org/10.1016/j.jallcom.2007.04.032.

(33) Hasegawa, S.; Sugimoto, T.; Hashimoto, T. Investigation of Structural Phase Transition Behavior of $\mathrm{SrZrO}_{3}$ by Thermal Analyses and High-Temperature X-Ray Diffraction. Solid State Ionics 
2010, 181 (23-24), 1091-1097. https://doi.org/10.1016/j.ssi.2010.06.035.

(34) Lanza, M.; Bersuker, G.; Porti, M.; Miranda, E.; Nafría, M.; Aymerich, X. Resistive Switching in Hafnium Dioxide Layers: Local Phenomenon at Grain Boundaries. Appl. Phys. Lett. 2012, 101, No. 193502.

(35) Zhang, H.; Yoo, S.; Menzel, S.; Funck, C.; Cueppers, F.; Wouters, D.J.; Hwang, C.S.; Waser, R.; Hoffmann-Eifert, S. Understanding the Coexistence of Two Bipolar Resistive Switching Modes with Opposite Polarity in $\mathrm{Pt} / \mathrm{TiO}_{2} / \mathrm{Ti} / \mathrm{Pt}$ Nanosized ReRAM Devices. ACS Appl. Mater. Interfaces. 2018, 10, 297766-29778. https://doi.org/10.1021/acsami.8b09068

(36) Shuai, Y.; Zhou, S.; Brger, D.; Helm, M.; Schmidt, H. Nonvolatile Bipolar Resistive Switching in $\mathrm{Au} / \mathrm{BiFeO}_{3} /$ Pt. J. Appl. Phys. 2011, 109 (12), 1-5. https://doi.org/10.1063/1.3601113.

(37) Chang, S. H.; Lee, J. S.; Chae, S. C.; Lee, S. B.; Liu, C.; Kahng, B.; Kim, D. W.; Noh, T. W. Occurrence of Both Unipolar Memory and Threshold Resistance Switching in a NiO Film. Phys. Rev. Lett. 2009, 102 (2), 1-4. https://doi.org/10.1103/PhysRevLett.102.026801.

(38) Chang, S. H.; Chae, S. C.; Lee, S. B.; Liu, C.; Noh, T. W.; Lee, J. S.; Kahng, B.; Jang, J. H.; Kim, M. Y.; Kim, D. W.; et al. Effects of Heat Dissipation on Unipolar Resistance Switching in Pt/NiO/Pt Capacitors. Appl. Phys. Lett. 2008, 92 (18), 28-31. https://doi.org/10.1063/1.2924304.

(39) Yang, Y. C.; Pan, F.; Zeng, F. Bipolar Resistance Switching in High-Performance Cu/ZnO:Mn/Pt Nonvolatile Memories: Active Region and Influence of Joule Heating. New J. Phys. 2010, 12. https://doi.org/10.1088/1367-2630/12/2/023008.

(40) Seo, S.; Lee, M.J.; Seo, D.H.; Jeoung, E.J.; Suh, D.-S.; Joung, Y.S.; Yoo, I.K.; Hwang, I.R.; Kim, S.H.; Byun, I.S.; Kim, J-S.; Choi, J.S.; Park, B.H. Reproducible Resistance Switching in Polycrystalline NiO Films. Appl. Phys. Lett. 2004, 85, 5655. https://doi.org/10.1063/1.1831560.

(41) He, L.; Liao, Z.-M.; Wu, H.-C.; Tian, X.-X.; Xu, D.-S.; Cross, G.L.W.; Duesberg, G.S.; Shvets, I.V.; Yu, D.-P. Memory and Threshold Resistance Switching in Ni/NiO Core-Shell Nanowires. Nano Lett. 2011, 11, 4601-4606. https://doi.org/10.1021/nl202017k.

(42) Peng, H.Y.; Li, Y.F.; Lin, W.N.; Wang, Y.Z.; Gao, X.Y.; Wu, T. Deterministic Converstion Between Memory and Threshold Resistive Switching via Tuning the Strong Electron Correlation. Sci. Rep. 2012, 2, 442. https://doi.org/10.1038/srep00442.

(43) Rupp, J.A.J.; Querré, M. ; Kindsmüller, A.; Besland, M.-P.; Janod, E.; Dittmann, R.; Waser, R.; Wouters, D.J. Different Threshold and Bipolar Resistive Switching Mechanisms in Reactively Sputtered Amorphous Undoped and Cr-Doped Vanadium Oxide Thin Films. J. Appl. Phys. 2018, 123, 044502. https://doi.org/10.1063/1.5006145.

(44) Diehm, P. M.; Ágoston, P.; Albe, K. Size-Dependent Lattice Expansion in Nanoparticles: Reality or Anomaly? ChemPhysChem 2012, 13 (10), 2443-2454. https://doi.org/10.1002/cphc.201200257.

(45) McCauley, D.; Newnham, R. E.; Randall, C. A. Intrinsic Size Effects in a Barium Titanate GlassCeramic. J. Am. Ceram. Soc. 2005, 81 (4), 979-987. https://doi.org/10.1111/j.11512916.1998.tb02435.x.

(46) Nian, Y. B.; Strozier, J.; Wu, N. J.; Chen, X.; Ignatiev, A. Evidence for an Oxygen Diffusion Model for the Electric Pulse Induced Resistance Change Effect in Transition-Metal Oxides. Phys. Rev. Lett. 2007, 98 (14), 3-6. https://doi.org/10.1103/PhysRevLett.98.146403.

(47) Das, A.; Gautam, S. K.; Shukla, D. K.; Singh, F. Correlations of Charge Neutrality Level with Electronic Structure and P-d Hybridization. Sci. Rep. 2017, 7 (January), 1-11. 
https://doi.org/10.1038/srep40843.

(48) Mete, E.; Shaltaf, R.; Ellialtıoglu. Electronic and Structural Properties of a 4d Perovskite: Cubic Phase of $\mathrm{SrZrO}_{3}$. Phys. Rev. B - Condens. Matter Mater. Phys. 2003, 68 (11), 3-6.

https://doi.org/10.1103/PhysRevB.68.035119.

(49) Akshay, V. R.; Arun, B.; Mandal, G.; Vasundhara, M. Visible Range Optical Absorption, Urbach Energy Estimation and Paramagnetic Response in Cr-Doped $\mathrm{TiO}_{2}$ Nanocrystals Derived by a SolGel Method. Phys. Chem. Chem. Phys. 2019, 21 (24), 12991-13004.

https://doi.org/10.1039/c9cp01351b.

(50) Harrigan, W. L.; Michaud, S. E.; Lehuta, K. A.; Kittilstved, K. R. Tunable Electronic Structure and Surface Defects in Chromium-Doped Colloidal $\mathrm{SrTiO}_{3-\delta}$ Nanocrystals. Chem. Mater. 2016, 28 (2), 430-433. https://doi.org/10.1021/acs.chemmater.6b00049.

(51) Jang, J. T.; Ko, D.; Ahn, G.; Yu, H. R.; Jung, H.; Kim, Y. S.; Yoon, C.; Lee, S.; Park, B. H.; Choi, S. J.; et al. Effect of Oxygen Content of the $\mathrm{LaAlO}_{3}$ Layer on the Synaptic Behavior of $\mathrm{Pt} / \mathrm{LaAlO}_{3} / \mathrm{Nb}$-Doped $\mathrm{SrTiO}_{3}$ Memristors for Neuromorphic Applications. Solid. State. Electron. 2018, 140 (October 2017), 139-143. https://doi.org/10.1016/j.sse.2017.10.032.

(52) Xu, Z. T.; Jin, K. J.; Gu, L.; Jin, Y. L.; Ge, C.; Wang, C.; Guo, H. Z.; Lu, H. Bin; Zhao, R. Q.; Yang, G. Z. Evidence for a Crucial Role Played by Oxygen Vacancies in $\mathrm{LaMnO}_{3}$ Resistive Switching Memories. Small 2012, 8 (8), 1279-1284. https://doi.org/10.1002/smll.201101796.

(53) Yang, Y.; Zhu, H.; Chu, D.; Liu, K.; Zhang, Y.; Pei, M.; Feng, S.; Jin, L.; Wang, C.; Liu, J.; et al. Modulation of the Resistive Switching of $\mathrm{BiFO}_{3}$ Thin Films through Electrical Stressing. J. Phys. D. Appl. Phys. 2020, 53 (11). https://doi.org/10.1088/1361-6463/ab5883.

(54) Oh, S.J.; Wang, Z.; Berry, N.E.; Choi, J.-H.; Zhao, T.; Gaulding, E.A.; Paik, T.; Lai, Y.; Murray, C.B.; Kagan, C.R. Engineering Charge Injection and Charge Transport for High Performance PbSe Nanocrystal Thin Film Devices and Circuits. Nano Lett. 2014, 14, 6210-6216. http://dx.doi.org/10.1021/nl502491d.

(55) Jang, S.; Son, D.; Hwang, S.; Kang, M.; Lee, S.-K.; Jeon, D.-Y.; Bae, S.; Lee, S.H.; Lee, D.S.; Kim, T.-W. Hybrid Dielectrics Composed of $\mathrm{Al}_{2} \mathrm{O}_{3}$ and Phosphonic Acid Self-Assembled Monolayers for Performance Improvement in Low Voltage Organic Field Effect Transistors. Nano Convergence 2018, 5, 20. https://doi.org/10.1186/s40580-018-0152-3.

(56) Tang, J.; Brzozowski, L.; Barkhouse, D.A.R.; Wang, X.; Debnath, R.; Wolowiec, R.; Palmiano, E.; Levina, L.; Pattantyus-Abraham, A.G.; Jamakosmanovic, D.; Sargent, E.H. Quantum Dot Photovoltaics in the Extreme Quantum Confinement Regime: The Surface-Chemical Origins of Exceptional Air- and Light-Stability. ACS Nano. 2010, 4, 869-878.

https://dx.doi.org/10.1021/nn901564q.

(57) Schmidt, D. O.; Hoffmann-Eifert, S.; Zhang, H.; La Torre, C.; Besmehn, A.; Noyong, M.; Waser, R.; Simon, U. Resistive Switching of Individual, Chemically Synthesized $\mathrm{TiO}_{2}$ Nanoparticles. Small 2015, 11 (48), 6444-6456.

(58) Waser, R.; Dittmann, R.; Staikov, G.; Szot, K. Redox-Based Resistive Switching Memories Nanoionic Mechanisms, Prospects, and Challenges. Adv. Mater. 2009, 21 (25-26), 2632-2663.

(59) Hou, J.; Nonnenmann, S. S.; Qin, W.; Bonnell, D. A. Size Dependence of Resistive Switching at Nanoscale Metal-Oxide Interfaces. Adv. Funct. Mater. 2014, 24 (26), 4113-4118.

(60) Gu, T. Role of Oxygen Vacancies in $\mathrm{TiO}_{2}$-Based Resistive Switches. J. Appl. Phys. 2013, 113 (3), 033707. 
(61) Chen, Y.; Chen, L.; Lian, G.; Xiong, G. Resistance and Superconductivity Switching Caused by Carrier Injection: Evidences of Self-Trapping Carriers in Oxide Electronics. J. Appl. Phys. 2009, $106(2), 023708$.

(62) Lampert, M.A. Volume-Controlled Current Injection in Insulators. Rep. Prog. Phys. 1964, 27, 329. https://doi.org/10.1088/0034-4885/27/1/307.

(63) Lim, E.W; Ismail, R. Conduction Mechanism of Valence Change Resistive Switching Memory: A Survey. Electronics 2015, 4, 586-613. https://doi.org/10.3390/electronics4030586.

(64) Desmond, K. W.; Weeks, E. R. Influence of Particle Size Distribution on Random Close Packing of Spheres. Phys. Rev. E - Stat. Nonlinear, Soft Matter Phys. 2014, 90 (2), 1-6. https://doi.org/10.1103/PhysRevE.90.022204.

(65) Yamada, S.; Kanno, J.; Miyauchi, M. Multi-Sized Sphere Packing in Containers: Optimization Formula for Obtaining the Highest Density with Two Different Sized Spheres. IPSJ Online Trans. 2011, 4, 126-133. https://doi.org/10.2197/ipsjtrans.4.126.

(66) Mulvaney, P.; Lees, E. E.; Nice, E. C.; Nguyen, T.-L.; Clayton, A. H. A.; Rothacker, J.; Gunzburg, M. J.; Howlett, G. J. Experimental Determination of Quantum Dot Size Distributions, Ligand Packing Densities, and Bioconjugation Using Analytical Ultracentrifugation. Nano Lett. 2008, 8 (9), 2883-2890. https://doi.org/10.1021/n1801629f.

(67) Yoko, A.; Wang, J.; Umezawa, N.; Ohno, T.; Oshima, Y. A-Site Cation Bulk and Surface Diffusion in A-Site-Deficient $\mathrm{BaZrO}_{3}$ and $\mathrm{SrZrO}_{3}$ Perovskites. J. Phys. Chem. C 2017, 121 (22), 12220-12229. https://doi.org/10.1021/acs.jpcc.7b02677.

(68) Yoko, A.; Akizuki, M.; Oshima, Y. Formation Mechanism of Barium Zirconate Nanoparticles under Supercritical Hydrothermal Synthesis. J. Nanoparticle Res. 2014, 16 (4), 1-9. https://doi.org/10.1007/s11051-014-2330-5.

(69) Rowberg, A. J. E.; Weston, L.; Van De Walle, C. G. Optimizing Proton Conductivity in Zirconates through Defect Engineering. ACS Appl. Energy Mater. 2019, 2 (4), 2611-2619. https://doi.org/10.1021/acsaem.8b02222.

(70) Youssef, M.; Van Vliet, K. J.; Yildiz, B. Polarizing Oxygen Vacancies in Insulating Metal Oxides under a High Electric Field. Phys. Rev. Lett. 2017, 119 (12), 1-6. https://doi.org/10.1103/PhysRevLett.119.126002. 
"For Table of Contents Only"
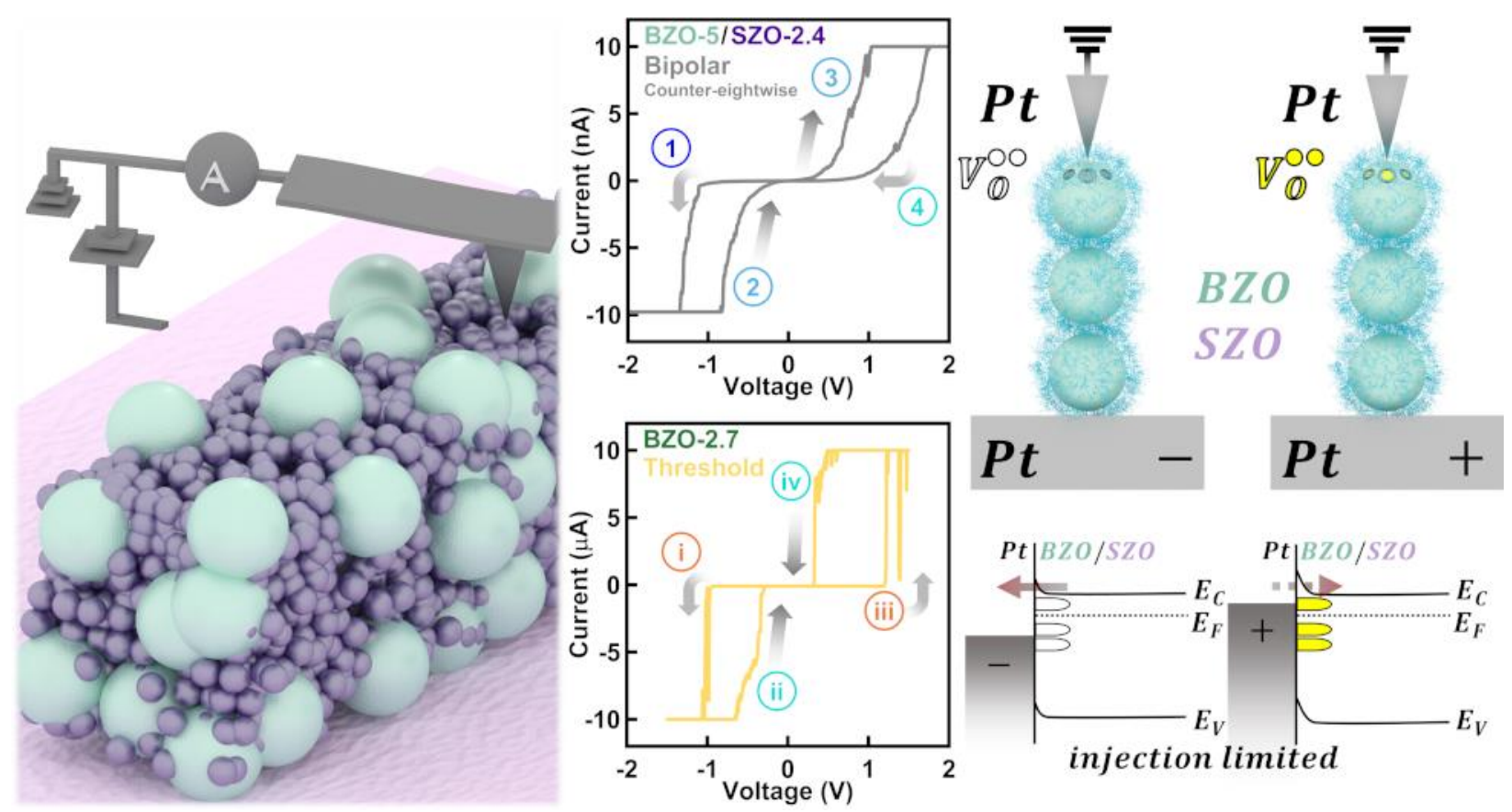